\title{
Closure to "Experimental Evaluation and Numerical Modeling of Wide-Flange Steel Columns Subjected to Constant and Variable Axial Load Coupled with Lateral Drift Demands"
}

\author{
Julien Cravero ${ }^{1}$, Ahmed Elkady ${ }^{2 *}$, Dimitrios G. Lignos ${ }^{3}$
}

The authors thank the discussers for their interest in the paper as well as their comments. The discussers raised a number of issues related to continuum finite element (CFE) simulation of steel wide-flange beam-columns. These issues are addressed in this closure based on pertinent literature and supplemental CFE simulations.

\section{Clarifications Regarding the Authors' Modelling Approach}

Before addressing the specific debate, the authors would like to clarify a few key issues with regards to their modelling approach that were not conveyed correctly by the discussers.

a) The discussers appear to mistakenly assume that the authors used an Explicit Solver to conduct the analysis reported in the paper. The authors used an Implicit Solver (ABAQUS Standard, v6.11) in Cravero et al. (2020) as well as prior related work (Elkady and Lignos 2015, 2018b) on steel wide-flange beam-column stability. The employed Implicit Solver involves Newton's method with double precision. The default convergence criteria of $0.5 \%$ on the relative force and moment residuals have been used.

b) The material model used in CFE simulations is the Voce and Chaboche constitutive law (Voce 1948; Armstrong and Frederick 1966; Chaboche 1989) available in ABAQUS/Standard with one backstress as reported in prior work by Elkady and Lignos (2018b). In that respect, it is not clear how the discussers computed the model parameters for a second backstress as stated in their discussion.

Considering all above, including other aspects of the model (i.e., use of rigid elements) the approach outlined and used by the discussers is not the same as that proposed and utilized by the authors. Notwithstanding these differences, the raised issues are addressed below in detail.

\section{Use of Local and/or Global Imperfections in Nonlinear Analysis of Wide-Flange Beam- Columns}

The discussers question the necessity to explicitly introduce local and/or global geometric imperfections (GIs) in CFE models to properly simulate the behavior of wide-flange beamcolumns under monotonic and cyclic loading. By simulating the response of one of the 12 specimens presented in Cravero et al. (2020) using ABAQUS and LS-DYNA, the discussers conclude that the authors' assertion that GIs generally need to be considered in CFE models, is overly conservative and software-dependent. The discussers attribute their conclusion to the

\footnotetext{
${ }^{1}$ Ph.D. Candidate, Hydrology Meteorology and Complexity (HMCO)| Navier, Ecole des Ponts ParisTech, 6-8 Ave. Blaise Pascal, 77420 Champs-sur-Marne, France. e-mail: julien.cravero@enpc.fr

${ }^{2}$ Lecturer (*Corresponding Author), Faculty of Engineering and Physical Sciences, University of Southampton, Burgess Rd., Boldrewood Innovation Campus, B178/4017, Southampton SO16 7QF, UK. e-mail: a.elkady@soton.ac.uk

${ }^{3}$ Associate Professor, École Polytechnique Fédérale de Lausanne (EPFL), GC B3 485, Station 18, 1015, Lausanne, Switzerland. e-mail: dimitrios.lignos@epfl.ch
} 
numerical precision of the ABAQUS solver which, unlike the LS-DYNA solver, does not appear to capture/trigger the geometric nonlinear effects, introduced by small deformations, in the first few elastic cycles, as the discussers observe in their LS-DYNA Explicit analyses.

As stated earlier, ABAQUS Implicit solver, with double-precision is what the authors have used throughout their studies on stability of beam-column members. In ABAQUS Explicit, double precision is mainly used for highly nonlinear problems, such as the one discussed in the paper. In fact, ABAQUS Explicit would warn against a single-precision run on the account of potentially large round-off errors, unless a large number of time increments (usually above 300k) are employed (see ABAQUS analysis user's guide, Section 6.1.2 v6.14 and onwards) (ABAQUS/Standard 2009). Moreover, based on Fig. 1a presented by the discussers, the column web out-plane displacement values are in the neighborhood of $0.05 \mathrm{~mm}$, which is substantially larger than the machine epsilon for single precision floating point numbers $\left(\epsilon=O\left(10^{-8}\right)\right)$. Accordingly, what the discussers claim to be the reason for the observed differences between the results of ABAQUS and LS-DYNA Explicit solvers is not related to the precision of a standard finite element program. If single precision were to be used with an Explicit solver, round-off errors in each step would accumulate and produce incorrect results. Of course, in an Implicit static analysis this is not an issue because the time step is much larger than that used in Explicit analysis. As such, round-off errors would not accumulate in this case given that the Implicit solver satisfies a convergence tolerance to compute the forward solution.

Considering that any CFE software (a) uses the same precision (double) and (b) explicitly considers a geometric nonlinearity transformation, the benchmark numerical solution for an "elastic" quasi-static finite element problem "shall" be that of the Implicit solver because unlike the Explicit one, it simply guarantees convergence between numerical solution steps. Moreover, significant spurious oscillations that can potentially arise in the numerical solution of an Explicit dynamic solver (Belytschko 1974; Maheo et al. 2011; Belytschko et al. 2014; Bathe 2019) will not occur. If both solvers are properly employed, then they should trace the same solution (at least for the elastic problem) as established by numerical analysis and finite element procedures. With the above aspects in mind, Fig. 1a herein illustrates that when a perfect geometry is used (i.e., no imposed GIs) the elastic solution (i.e., magnified response shown up to 200mm cumulative drift) as computed by the ABAQUS Implicit solver (noted as 'ABAQUS Implicit - Authors') does not contain any small deformations as observed in the discussers' prediction with the Explicit solver in LS-DYNA in the elastic loading phase. Referring to Fig. 1b, both the ABAQUS Explicit and Implicit solvers provide similar solutions even in the nonlinear regime. Only minor differences are observed at large inelastic deformations that are attributed to kinematic effects introduced in the Explicit analysis.

While the authors do not have access to specific (and important) details of the numerical models developed by the discussers (e.g., hourglass control for the employed shell element formulation, loading rates, time step, numerical damping, mass or time scaling, energy balance to ensure how small is the kinetic and viscous energies with respect to the internal energy, all this because of the use of the Explicit solver), it is believed that the initial small elastic out-of-plane web deformations that the discussers observe in their analysis with a perfect geometry (no GIs) are attributed to spurious oscillations that arise in the numerical solution of an Explicit dynamic solver (Belytschko 1974; Maheo et al. 2011; Belytschko et al. 2014; Rackauskaite et al. 2017; Bathe 2019). 
Particularly, CFE software, including LS-DYNA (Haufe et al. 2013; Jim et al. 2014; LS-DYNA 2017), highlight that these oscillations "shall" be eliminated from the numerical solution. If not, these oscillations, which are often referred to as "dynamic imperfections" (Rust and Schweizerhof 2003) could lead to erroneous simulated responses. The magnitude of these oscillations, as well as the numerical noise associated with Explicit solvers, is dependent on a number of parameters (some of which are listed above) including the finite element formulation and size, part interactions, boundary condition formulations, use of stiff elements, as well as the loading rates (i.e., it needs benchmarking). Most importantly, a CFE modeler has no systematic way to control the amplitude of spurious oscillations introduced by an Explicit solver because of their random nature. To that end, prior work with LS-DYNA (e.g., Rust and Schweizerhof 2003; Rackauskaite et al. 2017) as well as ABAQUS/Explicit (e.g., El Jisr et al. 2020) suggest carefully validated practices to properly benchmark an Explicit solver to address the above issues, particularly when dealing with quasi-static and/or a broad range of stability-sensitive problems. In the examined case (Specimen A4), these oscillations/imperfections are forgiving and seem to act as an alternative bucklingtrigger to explicitly modeled GIs within an Implicit solver environment as discussed in prior related work (Rust and Schweizerhof 2003).

Considering all above, the discussers do use imperfections in their analysis but these are of different nature than those suggested by the authors. As shown in this closure, these 'imperfections' are attributed to the Explicit solver rather than numerical precision considering the presented comparisons with the Implicit solver with double precision and the same geometric transformations within the CFE software.

\section{Final Considerations and Recommendations}

The authors think that global and/or local GIs as well as other model attributes (e.g., the inclusion of residual stresses) are certainly not always necessary to predict the behavior of wide-flange beam-columns depending on particular load and geometric configurations. For instance, a wellknown case in the context of this debate is the stability of stocky wide-flange members (Newell and Uang 2008) when attaining a stable equilibrium path even at large inelastic lateral drift demands (i.e., $6 \%$ rads) as well as cases in which high compressive axial load demands dominate the member response (Fell et al. 2009; Lamarche and Tremblay 2011). In the above two cases the role of local GIs is not likely to influence the member behavior. This conclusion, however, cannot be held in general.

Prior experimental work on the systematic characterization of the hysteretic behavior of wideflange beam-columns under multi-axial monotonic and cyclic loading across scales by the authors (Suzuki and Lignos 2015; Elkady and Lignos 2018a; Cravero et al. 2020) as well as other researchers (Newell and Uang 2008; Lamarche and Tremblay 2011; Cheng et al. 2013; Ozkula et al. 2017; Cheng et al. 2018) during the past decade demonstrate that the role of initial GIs (local and/or global) is substantiated in predictive CFE modelling of wide-flange beam-columns when these feature slender profiles and their hysteretic response is dominated by early onset of local buckling or by coupled member and local geometric instabilities. The significance of accurate steel material modelling has also been stressed. The above findings have also been corroborated by results of two blind analysis competitions that were organized a few years ago by NIST-ATC (2018) to predict the cyclic behavior of deep wide-flange beam-columns. 
The authors propose a systematic modelling approach that may be used for conventional continuum finite element analysis to assess a member's stability. While this approach may not be the only one available in the literature, each one of its main 'ingredients' are clearly defined and quantified in prior work; most importantly they are FE-platform independent, and they are consistent with structural stability concepts (Galambos 1998) as well as observations from physical experiments. In brief, this approach includes:

a) A rate-independent multiaxial plasticity model along with a systematic methodology to tackle the well-known model parameter non-uniqueness (de Castro e Sousa et al. 2020).

b) Quantitative residual stress patterns due to hot rolling (Sousa and Lignos 2017) that may not necessarily be important for yield or peak strength predictions but are certainly important when it comes to torsional stiffnesses of wide-flange cross-sections due to their influence on the Wagner constant (Trahair 1993).

c) Quantitative introduction of initial GIs to properly simulate the onset of nonlinear geometric instabilities in beam-columns undergoing a softening equilibrium path (Elkady and Lignos 2018b). Given a) and b) above, the initial GIs (shape and magnitude) represent the "end product" that a modeler attempts to idealize within the CFE software including welded base plates and fabrication work. The use of GIs in nonlinear analysis is consistent with core concepts of structural stability (Galambos 1998), which are also included in our design standards when it comes to stability verification of structural members (AISC 2016; Ziemian et al. 2018).

d) The use of a nonlinear geometric transformation as well as an Implicit solver with the tolerance characteristics discussed earlier. For standard 'member' stability verification, which is the particular problem under question herein, the use of Implicit solvers is recommended simply because an Explicit solver comes with a number of challenges to overcome. For the problem in question i.e., member stability verification, the computational cost by using an Implicit solver is not prohibitive even with a personal computer.

With regard to discussers' final point involving the practicality of assigning GIs into members within a system-level simulation, the authors are of the opinion that this existent difficulty can be comfortably overcome through systematic rules to assign GIs at the member level. Particularly, at the system level, GIs can be defined to each member during the assembly of the CFE model by using scripting tools such as Python that can be seamlessly integrated into any commercial CFE. In this case, for instance, local GIs could be based on conventional plate buckling theory rather than buckling eigenvalue analysis. However, the use of spurious oscillations attributed to the Explicit solver is not recommended for this or any other purpose. 


\section{References}

ABAQUS/Standard (2009). User's Manual, Version 6.14, Dassault Systèmes Simulia Corp, Providence, RI.

AISC (2016). "Specification for structural steel buildings." ANSI/AISC 360-16, Chicago, IL.

Armstrong, P. J., and Frederick, C. O. (1966). "A mathematical representation of the multiaxial bauschinger effect." Materials at High Temperatures, 24(1), 1-26, DOI: 10.1179/096034007X207589.

Bathe, K.-J. (2019). Finite element procedures, $2^{\text {nd }}$ Edition, Prentice Hall, Pearson Education, Inc., New Jersey, USA.

Belytschko, T. (1974). "Transient analysis (computer programs and algorithms)." Structural Mechanics Computer Programs: Surveys, Assessments, and Availability, 255-276, DOI:

Belytschko, T., Liu, W. K., Moran, B., and Elkhodary, K. I. (2014). Nonlinear finite elements for continua and structures, $2^{\text {nd }}$ Edition. John Wiley \& Sons, Inc., New Jersey, United States

Chaboche, J.-L. (1989). "Constitutive equations for cyclic plasticity and cyclic viscoplasticity." International journal of plasticity, 5(3), 247-302, DOI: 10.1016/0749-6419(89)90015-6

Cheng, X., Chen, Y., and Nethercot, D. A. (2013). "Experimental study on h-shaped steel beam-columns with large width-thickness ratios under cyclic bending about weak-axis." Engineering Structures, 49, 264-274, DOI: 10.1016/j.engstruct.2012.10.035

Cheng, X., Chen, Y., Niu, L., and Nethercot, D. A. (2018). "Experimental study on h-section steel beamcolumns under cyclic biaxial bending considering the effect of local buckling." Engineering Structures, 174, 826-839, DOI: 10.1016/j.engstruct.2018.08.001.

Cravero, J., Elkady, A., and Lignos, D. G. (2020). "Experimental evaluation and numerical modeling of wide-flange steel columns subjected to constant and variable axial load coupled with lateral drift demands." Journal of Structural Engineering, 146(3), DOI: 10.1061/(ASCE)ST.1943$541 X .0002499$.

de Castro e Sousa, A., Suzuki, Y., and Lignos, D. (2020). "Consistency in solving the inverse problem of the voce-chaboche constitutive model for plastic straining." Journal of Engineering Mechanics, 146(9), 04020097, DOI: 10.1061/(ASCE)EM.1943-7889.0001839.

El Jisr, H., Elkady, A., and Lignos, D. G. (2020). "Hysteretic behavior of moment-resisting frames considering slab restraint and framing action." ASCE Journal of Structural Engineering, 146(8), DOI: 10.1061/(ASCE)ST.1943-541X.0002696.

Elkady, A., and Lignos, D. G. (2015). "Analytical investigation of the cyclic behavior and plastic hinge formation in deep wide-flange steel beam-columns." Bull Earthquake Eng, 13(4), 1097-1118, DOI: 10.1007/s10518-014-9640-y.

Elkady, A., and Lignos, D. G. (2018a). "Full-scale testing of deep wide-flange steel columns under multiaxis cyclic loading: Loading sequence, boundary effects and out-of-plane brace force demands." ASCE Journal of Structural Engineering, 144(2), 04017189, DOI: 10.1061/(ASCE)ST.1943$541 X .0001937$.

Elkady, A., and Lignos, D. G. (2018b). "Improved seismic design and nonlinear modeling recommendations for wide-flange steel columns." ASCE Journal of Structural Engineering, 144(9), 04018162, DOI: 10.1061/(ASCE)ST.1943-541X.0002166.

Fell, B. V., Kanvinde, A. M., Deierlein, G. G., and Myers, A. T. (2009). "Experimental investigation of inelastic cyclic buckling and fracture of steel braces." Journal of structural engineering, 135(1), 1932, DOI: 10.1061/(ASCE)0733-9445(2009)135:1(19).

Galambos, T. V. (1998). Technical memoranda of structural stability research council, 5, John Wiley \& Sons, Inc., New Jersey, United States.

Haufe, A., Schweizerhof, K., and DuBois, P. (2013). "Properties \& limits: Review of shell element formulations." Proc., The 2013 LS-Dyna Developer Forum, Filderstadt (Stuttgart), Germany.

Jim, D., Philip, H., and Paul, D. B. (2014). "LS-DYNA analysis for structural mechanics." Finite Element Analysis, Predictive Engineering, Portland, Oregon, USA. 
Lamarche, C.-P., and Tremblay, R. (2011). "Seismically induced cyclic buckling of steel columns including residual-stress and strain-rate effects." Journal of Constructional Steel Research, 67(9), 1401-1410, DOI: $10.1016 / \mathrm{j} . j \mathrm{csr} .2010 .10 .008$.

LS-DYNA (2017). "Theory manual." Livermore Software Technology Corporation (LSTC), Livermore, CA, USA, 882.

Maheo, L., Rio, G., and Grolleau, V. (2011). "On the use of some numerical damping methods of spurious oscillations in the case of elastic wave propagation." Mechanics Research Communications, 38(2), 81-88, DOI: 10.1016/j.mechrescom.2011.01.006.

Newell, J. D., and Uang, C.-M. (2008). "Cyclic behavior of steel wide-flange columns subjected to large drift." Journal of structural engineering, 134(8), 1334-1342, DOI: 10.1061/(ASCE)07339445(2008)134:8(1334).

NIST-ATC (2018). "Atc-106-1 beam column blind prediction contest." < https://www.atcouncil.org/atc106-blind-contest>.

Ozkula, G., Harris, J., and Uang, C. M. (2017). "Observations from cyclic tests on deep, wide-flange beamcolumns." AISC Engineering Journal, 54(1), 45-61.

Rackauskaite, E., Kotsovinos, P., and Rein, G. (2017). "Model parameter sensitivity and benchmarking of the explicit dynamic solver of 1s-dyna for structural analysis in case of fire." Fire Safety Journal, 90, 123-138, DOI: 10.1016/j.firesaf.2017.03.002.

Rust, W., and Schweizerhof, K. (2003). "Finite element limit load analysis of thin-walled structures by ansys (implicit), 1s-dyna (explicit) and in combination." Thin-walled structures, 41(2-3), 227-244, DOI: $10.1016 / \mathrm{S} 0263-8231(02) 00089-7$.

Sousa, A. A., and Lignos, D. G. (2017). "Residual stress measurements of european hot-rolled i-shaped steel profiles." EPFL-REPORT-231302, Ecole Polytechnique Federale de Lausanne (EPFL), Lausanne, Switzerland.

Suzuki, Y., and Lignos, D. G. (2015). "Large scale collapse experiments of wide-flange steel beamcolumns." Proc., $8^{\text {th }}$ International Conference on Behavior of Steel Structures in Seismic Areas, Shanghai, China.

Trahair, N. S. (1993). Flexural-torsional buckling of structures, CRC Press, Boca Raton, Florida, United States.

Voce, E. (1948). "The relationship between dtress and strain for homogeneous deformation." Journal of the Institute of Metals, 74, 537-562.

Ziemian, R. D., B., A. J. C., Denavit, M. D., and Denavit, T.-J. L. (2018). "Three-dimensional benchmark problems for design by advanced analysis: Impact of twist." Journal of Structural Engineering, 144(12), 04018220, DOI: 10.1061/(ASCE)ST.1943-541X.0002224. 
257

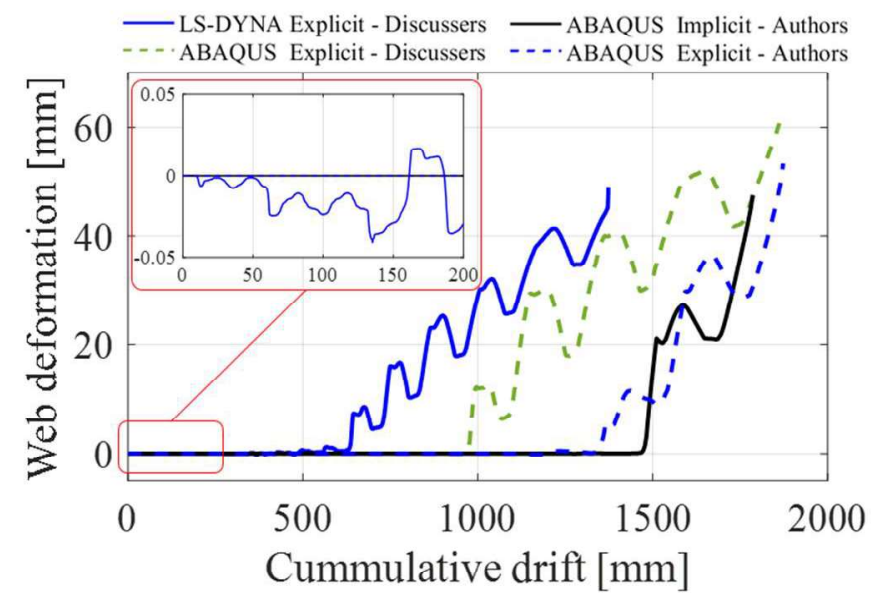

(a)

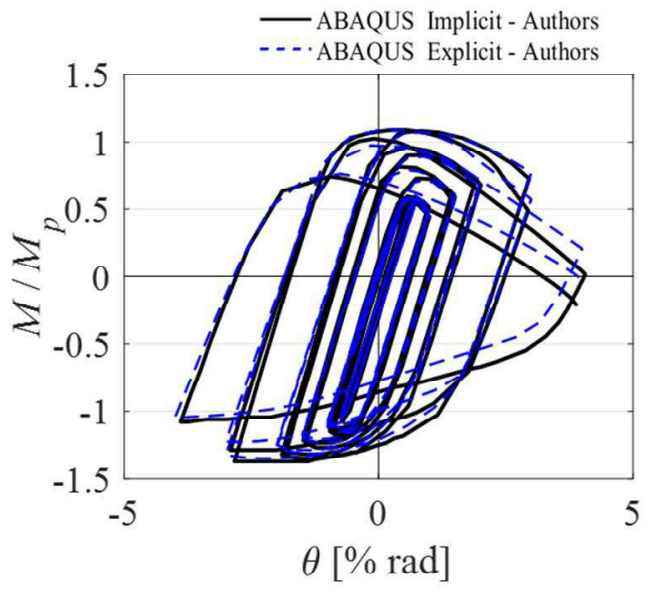

(b) 